\title{
Nonintubated uniportal thoracoscopic wedge resection for early lung cancer
}

\author{
Tung-Ming Tsai, Mong-Wei Lin, Hsao-Hsun Hsu, Jin-Shing Chen \\ Department of Surgery, National Taiwan University Hospital and National Taiwan University College of Medicine, Taipei, Taiwan \\ Contributions: (I) Conception and design: JS Chen; (II) Administrative support: JS Chen, HH Hsu; (III) Provision of study materials or patients: JS \\ Chen, HH Hsu, MW Lin; (IV) Collection and assembly of data: TM Tsai; (V) Data analysis and interpretation: JS Chen, TM Tsai; (VI) Manuscript \\ writing: All authors; (VII) Final approval of manuscript: All authors. \\ Correspondence to: Jin-Shing Chen, MD, PhD. Department of Surgery, National Taiwan University Hospital and National Taiwan University College \\ of Medicine, No. 7, Chung-Shan South Road, Taipei 10002, Taiwan. Email: chenjs@ntu.edu.tw.
}

\begin{abstract}
Background: Minimal invasive surgery is current choice of treatment for lung cancer. Combined nonintubated anesthesia with uniportal thoracoscopic surgery is not well understood. Here, we report the experience of nonintubated uniportal thoracoscopic surgery in the treatment of primary non-small cell lung cancer (NSCLC).
\end{abstract}

Methods: From January 2014 to December 2015, we retrospectively reviewed 131 consecutive patients with primary NSCLC who underwent nonintubated uniportal thoracoscopic wedge resection and mediastinal lymph node dissection at a single medical center.

Results: Of the 131 patients, 110 (84\%) received preoperative computed tomography-guided dye localization. Most of them were diagnosed with early stage invasive adenocarcinoma ( $\mathrm{N}=112,85.5 \%$; pathological stage IA: $84.7 \%, \mathrm{~N}=111$ ), and the mean size of the nodule was small (diameter: $0.85 \pm 0.40 \mathrm{~cm}$ ). All section margins were free of malignancy. In total, 7 of the 131 patients $(5.3 \%)$ had their treatment converted from uniportal to multi-portal video-assisted thoracoscopic surgery (VATS), and 1 (0.8\%) had his treatment converted to endotracheal intubation with general anesthesia. The mean operation time was $91.1 \pm 32.6$ minutes, and the postoperative complications included pneumonia $(0.8 \%)$, prolonged air leaks (0.8\%), and subcutaneous emphysema (1.5\%).

Conclusions: Overall, nonintubated uniportal VATS is a feasible, effective and safe procedure for the treatment of early primary lung cancer.

Keywords: Primary non-small cell lung cancer; nonintubated surgery; uniportal video-assisted thoracoscopic
surgery (uniportal VATS); preoperative computed tomography-guided dye localization

Received: 31 July 2017; Accepted: 24 August 2017; Published: 06 November 2017.

doi: 10.21037/jovs.2017.08.21

View this article at: http://dx.doi.org/10.21037/jovs.2017.08.21

\section{Introduction}

Lung cancer has been one of the most serious public health concerns for many decades due to its high prevalence and mortality (1). Non-small cell lung cancer (NSCLC) is the most common form of lung cancer, and surgical resection is the gold standard of curative treatment. The National Lung Screening Trial, initiated in 2002, revealed that lowdose computed tomography (LDCT) screening in high-risk patients can reduce the lung cancer mortality significantly and has enabled a higher proportion of lung cancers to be diagnosed at an early stage (2). Thus, thoracic surgeons today are treating smaller pulmonary nodules than before. An ongoing challenge, however, is determining how to minimize patients' surgical trauma with less invasive procedures; surgeons are also focused on adopting a more lung-sparing pulmonary resection to improve lung reservation. 


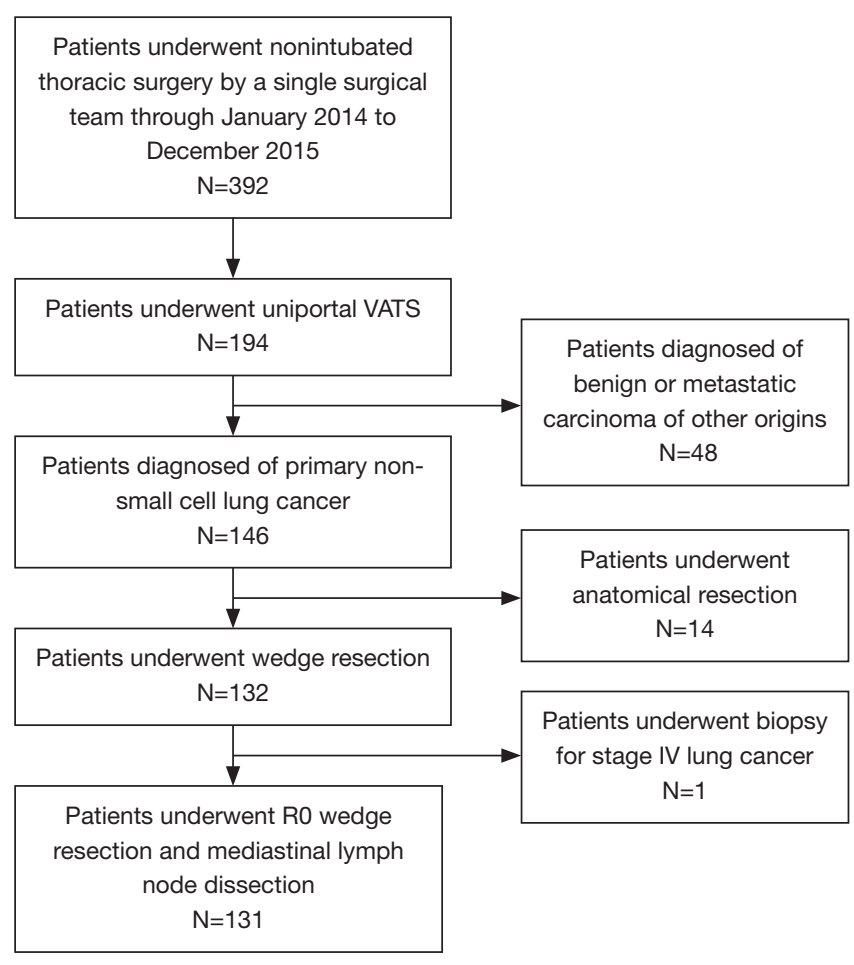

Figure 1 Algorithm for patient selection. CT, computed tomography; VATS, video-assisted thoracoscopic surgery.

Video-assisted thoracic surgery (VATS) is a revolutionary technique that has emerged over the past two decades. VATS for pulmonary resection has already proven its safety, feasibility, and ability enhance surgical outcome compared with traditional thoracotomy, especially in the treatment of primary lung cancer (3). One recent advancement in VATS has been the introduction of the uniportal technique, which was first reported on in 2004 for cases of pulmonary wedge resections (4). Compared with traditional VATS, which usually requires three or four entry ports, the uniportal technique requires only a single incision (length: 3 to $4 \mathrm{~cm}$ ) in the intercostal space; this increase patients' comfort and efficient recovery (5). Moreover, nonintubated anesthesia is not only safe and feasible, but also reduces the adverse effects associated with trachea intubation and general anesthesia (6-8). Substantial progress has been made in developing and improving VATS based on these two methods.

Since early 2014, we have been combining these two innovations, the uniportal technique and nonintubated anesthesia, into VATS for the management of lung lesions (9-11). We reported our first experience with 116 patients who underwent either wedge resections or anatomical resections, revealing that the nonintubated uniportal thoracoscopic surgery is a feasible and safe procedure, especially for those with small $(\leq 20 \mathrm{~mm})$ and peripheral lung lesions. This successful result encouraged us to utilize this technique to treat different types of surgical candidates, particularly those with malignant pulmonary disease. In the current study, we evaluated the clinical outcomes of using the nonintubated uniportal thoracoscopic wedge resection to treat patients with primary NSCLC.

\section{Methods}

\section{Study designs and patients}

The medical records of 131 consecutive patients who underwent nonintubated uniportal thoracoscopic wedge resection for primary NSCLC by a single thoracic surgical team using the same clinical protocols, care patterns, and perioperative orders at National Taiwan University Hospital from January 2014 to December 2015 were reviewed retrospectively. During this period, 392 patients received nonintubated thoracic surgery, including 194 patients who underwent nonintubated uniportal VATS (146 cases were diagnosed with primary NSCLC in their final pathology reports). Figure 1 shows the algorithm that was used to select the patients for this study. Approval for this study was granted by the National Taiwan University Hospital Research Ethics Committee (project approval number: 201706092RINA).

We collected the patients' clinical parameters, including age, sex, smoking status, underlying diseases, nodule size, nodule location, invasive procedure, operation time, final pathology report, postoperative chest tube drainage duration and hospital stay from their medical charts. The histopathology of the resected nodules was defined by matching the patients' data with criteria published by the World Health Organization in 2015 (12).

Based on our experience and publications since August 2009 (6-10,13-15), the appropriate selection criteria for nonintubated VATS comprise the following: (I) the primary tumor size should not exceeded $6 \mathrm{~cm}$ in diameter and (II) there should be no evidence of direct invasion into the chest wall, diaphragm or main bronchus. However, patients with an American Society of Anesthesiologists score $\geq 4$, sleep apnea, unfavorable airway, or a spinal anatomy or chest wall deformity should be excluded. The selection of patients who underwent uniportal VATS was made according to 
the judgment of the thoracic surgeons, who considered clinical information such as tumor size and location via the preoperative CT image.

\section{Preoperative CT-guided dye localization}

Surgeons carefully evaluate the patients' tumor size, localization, and appearance in their final preoperative CT image. If the tumor is small, located far from the lung surface, or predominantly composed of groundglass component, it is difficult to localize the tumor intraoperatively, especially in a uniportal thoracoscopic setting. Therefore, a preoperative CT-guided patent blue vital (PBV) dye localization technique, the details of which we described elsewhere, was arranged under the consensus of both surgeon and radiologist (16). The dye localization was performed preoperatively by experienced radiologists. We adopted low-dose settings $(120 \mathrm{kVp}, 50 \mathrm{~mA}, 1.3$ pitch, and 0.7 $\mathrm{s}$ rotation time) without contrast enhancement on a 16-slice instrument (GE LightSpeed; GE Healthcare, Milwaukee, WI, USA), and laid the patients in a suitable position that enabled the shortest access route for needle insertion. Under local anesthesia (xylocaine 2\%; Recipharm Monts, Monts, France) at the puncture site, a 22-gauge Chiba needle was inserted toward to the deep parts of the nodule. A total of $0.2 \mathrm{~mL}$ of PBV dye (patent blue V 2.5\%; Guerbet, Aulnaysous-Bois, France) contained in a $1-\mathrm{mL}$ syringe was injected into the nodule along the needle tract and at the subpleural parenchyma. Once the procedure was complete, the patients were transferred to the operating room to await surgery.

\section{Nonintubated anesthetic setting, induction and sedation}

We have described the protocol of nonintubated VATS surgery in full in our previous publications (6-10,13-15). In brief, the patients were sedated with intravenous fentanyl (50-100 mg) and continuously monitored by electrocardiography, pulse oximetry, arterial blood pressure, and respiratory rate. A detector for measuring the end tidal carbon dioxide $\left(\mathrm{ETCO}_{2}\right)$ was inserted into one nostril in each patient, and a bispectral index (BIS) sensor (BIS Quatro; Aspect Medical System, Norwood, MA, USA) was employed to constantly monitor the patients' consciousness during anesthesia. During the operation, the patients were maintained under sedation by using a target-controlled infusion method with intravenous propofol, and were laid in the lateral decubitus position. All the patients breathed spontaneously through an oxygen mask and retained an oxygen saturation of $\mathrm{SpO}_{2}>90 \%$.

\section{Surgical setting of the uniportal VATS}

As noted, all the patients were laid in the full lateral decubitus position with a slight flexion of the table at mid-chest level. Under local anesthesia with $2 \%$ lidocaine, a single skin incision (length: $3 \mathrm{~cm}$ ) was made at the fifth intercostal space along the anterior axillary line to create a working port. We then dissected along the working port into the thoracic cavity to create an iatrogenic open pneumothorax. Once the lung collapsed, the working port was covered by a wound protector (Alexis wound protector/retractor; Applied Medical Technology, Brecksville, OH, USA) that enabled easy access for the instruments. A 5-mm, 30-degree video telescope (Karl Storz, Tuttlingen, Germany) was placed in the working port to explore the pleural cavity.

\section{Intercostal and vagal nerve block}

Under the guidance of a 5-mm thoracoscope, the intercostal and vagal nerve block was performed via direct vision. Using a $25-\mathrm{G}$ top-winged infusion needle, $0.5 \%$ bupivacaine (1.5 $\mathrm{mL}$ for each intercostal space) was applied from the third to the eighth intercostal nerve, $2 \mathrm{~cm}$ lateral to the sympathetic chain $(14,15)$. The intercostal nerve block contributes to analgesic control during the entire operation. In addition, the intrathoracic vagal block is routinely used to reduce the coughing reflex during lung manipulations $(6,15)$. Three milliliters of bupivacaine was then injected via a long-needle instrument into the vagus nerve near the lower paratrachea (for right-sided procedures) and the aortopulmonary window (for left-sided procedures). Notably, both the intercostal and vagal blocks can be repeated in prolonged operations if necessary.

\section{Wedge resection techniques in uniportal VATS}

The nodule can be localized through several ways, including direct vision, instrument palpitation or preoperative PBV dye localization. Here, after confirming the site of the lesion, we used two 2-0 silk anchoring retraction sutures to lift up the nodule (17). By using a long needle to penetrate the chest wall, the retraction sutures were pulled extra corporeally in the optimal direction for wedge resection with an endoscopic stapling device. The resected lungs were then removed with an endoscopic retrieval bag, followed by a systematic mediastinal lymph node dissection using a 


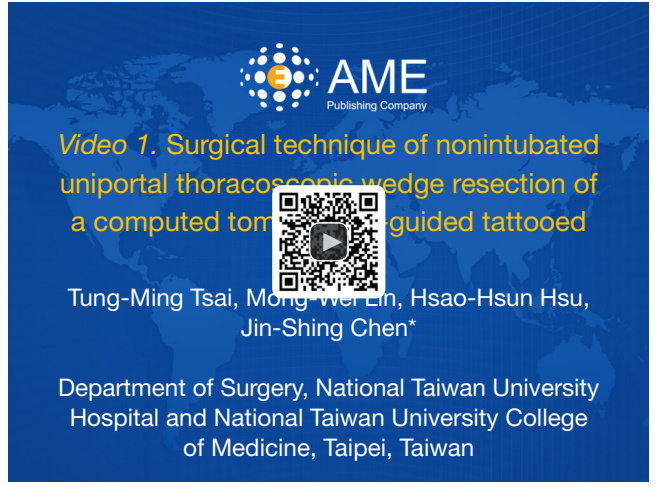

Figure 2 Surgical technique of nonintubated uniportal thoracoscopic wedge resection of a computed tomography-guided tattooed lesion (18). Available online: http://www.asvide.com/manuscripts/1769

harmonic scalpel (Ethicon Endo-Surgery, Cincinnati, OH, USA) and endoscopic electrocautery. A chest tube (24or 28-French) or a small-bore pigtail catheter (8-French; Bioteque Corporation, I-Lan, Taiwan) was inserted through the working port, followed by a manually positive-pressure mask ventilation to expand the collapsed operated lungs to check for the presence of air leaks. At the end of surgery, the patients were fully awake and able to breathe deeply and cough independently. The complete operative procedures are demonstrated in the Figure 2.

\section{Conversion to multi-portal VATS or tracheal intubation}

In cases of an unidentified nodule, an inadequate section margin, a complicated anatomical dissection, or unexpected pleural adhesions, the surgeon may decide to add extra working ports intraoperatively. The indications for conversion to tracheal intubation during nonintubated VATS include: profound respiratory movement, massive pleural adhesions, persistent hypoxemia (oxygen saturation $<80 \%$ ), unstable hemodynamic status, or uncontrolled bleeding requiring an emergency thoracotomy $(6-8,13,19)$. The details of the conversion procedure have been described previously (14). In brief, we first inserted a chest tube through the working port, then covered the surgical wounds with transparent waterproof dressings (Tegaderm Film; 3M Health Care, Neuss, Germany), resulting in a reexpansion of the collapsed lung. Next, the anesthesiologist performed endotracheal intubation under the guidance of a bronchoscope and used a bronchial blocker for lung separation. During the entire procedure, the patient was kept in the fully lateral decubitus position without change. The operation immediately resumed once the endotracheal intubation was complete.

\section{Postoperative patient care}

In general, all the patients in this study were managed postoperatively according to the same protocol. The patients were able to resume oral intake, and were encouraged to engage in early ambulation, $2 \mathrm{~h}$ after their surgery. We used oral nonsteroidal analgesics $(325 \mathrm{mg}$ of acetaminophen and $37.5 \mathrm{mg}$ of tramadol) combined with patient-controlled analgesia with intravenous morphine $(1 \mathrm{mg} / \mathrm{mL})$ for the first 72 hours for pain control. A postoperative chest radiograph was obtained immediately after surgery or the following morning. The chest tube was removed if there was no ongoing air leak and if drainage was $<200 \mathrm{~mL}$ per $24 \mathrm{~h}$. Notably, prolonged air leakage was defined as persistent air leakage for more than 3 days after the operation. All postoperative complications requiring medication or intervention were recorded.

\section{Outcome variables and statistical analysis}

Continuous variables, such as age and body weight, are presented in this paper as mean \pm standard deviation. A two-sample t-test or Mann-Whitney U test was used for the statistical analysis. By contrast, categorical variables, such as sex and smoking status, are presented herein as frequencies (\%), and a Pearson's chi-squared test or Fisher exact test was used for the analysis. All data analyses were conducted using SPSS Version 22 (SPSS Inc.; IBM, Chicago, IL, USA) and statistical significance was set at $\mathrm{P}<0.05$.

\section{Results}

Table 1 presents a list of the clinical data of the 131 patients enrolled in this study. The mean age of the patients was $57.6 \pm 10.9$ years (range: $26-84$ years). Most of them were female with an average physique and adequate lung capacity; only a few $(3.8 \%)$ were current smokers. Some of the patients had a comorbid disease, such as cardiac disease (14.5\%), hypertension $(26.7 \%)$, or extrathoracic malignancy of other origins $(15.3 \%)$. One patient $(0.8 \%)$ had received ipsilateral lung surgery preoperatively due to recurrent pneumothorax.

Table 2 reveals the surgical and postoperative outcomes. All the patients received nonintubated uniportal VATS wedge resection of the lungs and mediastinal lymph 
Table 1 Patients' demographic data $(\mathrm{N}=131)$

\begin{tabular}{lc}
\hline Variable & $\mathrm{N}(\%)$ or mean \pm SD (range) \\
\hline Age (years) & $57.6 \pm 10.9[26-84]$ \\
Sex (female) & $113(86.3)$ \\
Height (cm) & $158.9 \pm 7.7(140.3-179.2)$ \\
Weight (kg) & $56.9 \pm 9.5(34-86.3)$ \\
BMI & $22.5 \pm 3.0(13.7-32.0)$ \\
Current smokers & $5(3.8)$ \\
FVC (\%) & $107.9 \pm 14.6(78.6-141.2)$ \\
FEV $1 \%$ ) & $107.8 \pm 17.7(48.7-172.1)$ \\
CEA (ng/mL) & $1.56 \pm 0.91(0.1-6.07)$ \\
Comorbidity & $5(3.8)$ \\
COPD or asthma & $19(14.5)$ \\
Cardiac disease & $35(26.7)$ \\
Hypertension & $8(6.1)$ \\
Diabetes mellitus & $4(3.1)$ \\
Cerebrovascular accident & $20(15.3)$ \\
Extra-thoracic malignancy & $1(0.8)$ \\
Previous ipsilateral lung surgery &
\end{tabular}

BMI, body mass index; FVC, forced vital capacity. FEV ${ }_{1}$, forced expiratory volume in $1 \mathrm{~s}$; CEA, carcinoembryonic antigen; COPD, chronic obstructive pulmonary disease.

node dissection. The mean duration of operation was $91.1 \pm 32.6 \mathrm{~min}$ (range, $41-235 \mathrm{~min}$ ). Eight of the patients required intraoperative conversion from nonintubated uniportal surgery to intubated or multi-portal VATS. One patient's $(0.8 \%)$ operation was converted to endotracheal intubation with general anesthesia because of severe pleural adhesions and strong mediastinal movement; the other 7 patients' $(5.3 \%)$ operations were converted to multi-portal VATS. Among these seven patients, three conversions were performed by using an additional accessory port to find the nodule via finger palpitation: in two patients without preoperative PBV dye localization, the nodules were too faint and small and prevented identification with instruments and direct vision; in the other patient, we could not identify the PBV dye because the nodule was located too deeply. In the remaining four conversions, two patients' operations were converted due to severe mediastinal or pulmonary adhesions, one was converted was due to vigorous diaphragm movement, and one was converted
Table 2 Surgical results and postoperative outcome $(\mathrm{N}=131)$

\begin{tabular}{|c|c|}
\hline Variable & $\mathrm{N}(\%)$ or mean $\pm \mathrm{SD}$ (range) \\
\hline $\begin{array}{l}\text { Preoperative CT-guided PBV } \\
\text { localization }\end{array}$ & $110(84.0)$ \\
\hline Operative time (min) & $91.1 \pm 32.6[41-235]$ \\
\hline Nodule size $(\mathrm{cm})$ & $0.85 \pm 0.40(0.2-3.2)$ \\
\hline \multicolumn{2}{|l|}{ Histology of each nodule } \\
\hline Invasive adenocarcinoma & $112(85.5)$ \\
\hline Adenocarcinoma in situ & $17(13.0)$ \\
\hline $\begin{array}{l}\text { Lymphoepithelioma-like } \\
\text { carcinoma }\end{array}$ & $1(0.8)$ \\
\hline Carcinoid, typical & $1(0.8)$ \\
\hline \multicolumn{2}{|l|}{ Pathological stage } \\
\hline 0 & $17(13.0)$ \\
\hline IA & $111(84.7)$ \\
\hline IB & $3(2.3)$ \\
\hline Blood loss (mL) & $27.2 \pm 16.1(0-100)$ \\
\hline \multicolumn{2}{|l|}{ Conversion } \\
\hline Trachea intubation & $1(0.8)$ \\
\hline Multi-ports VATS & $7(5.3)$ \\
\hline Thoracotomy & 0 \\
\hline Chest tube drainage (days) & $1.4 \pm 1.3(0-7)$ \\
\hline $\begin{array}{l}\text { Post-operative hospital stay } \\
\text { (days) }\end{array}$ & $3.3 \pm 1.4[1-10]$ \\
\hline \multicolumn{2}{|l|}{ Complications } \\
\hline Subcutaneous emphysema & $2(1.5)$ \\
\hline Prolong air-leaks $>3$ days & $1(0.8)$ \\
\hline Pneumonia & $1(0.8)$ \\
\hline 30-day mortality & 0 \\
\hline
\end{tabular}

CT, computed tomography; VATS, video-assisted thoracoscopic surgery.

to achieve an adequate section margin. The pathological reports revealed most of the patients were diagnosed with early stage (stage IA, $84.7 \%$ ) invasive adenocarcinoma $(\mathrm{N}=112,85.5 \%)$. Overall, the postoperative course was smooth. Most of the patients required chest tube drainage for 1 day and were discharged 3 to 4 days after the operation. Postoperative complications were reported in 4 of the patients: 1 had prolonged air leaks, 1 developed pneumonia, and 2 developed subcutaneous emphysema. 
Table 3 Results of preoperative CT-guided PBV dye localization $(\mathrm{N}=110)$

\begin{tabular}{|c|c|}
\hline Variable & $\mathrm{N}(\%)$ or mean $\pm \mathrm{SD}$ (range) \\
\hline Trans-lung needle length $(\mathrm{cm})$ & $1.95 \pm 1.23(0.3-6.3)$ \\
\hline \multicolumn{2}{|l|}{ Lobes of localization } \\
\hline Left upper lobe & $33(30.0)$ \\
\hline Left lower lobe & $14(12.7)$ \\
\hline Right upper lobe & $35(31.8)$ \\
\hline Right middle lobe & $6(5.5)$ \\
\hline Right lower lobe & $22(20.0)$ \\
\hline \multicolumn{2}{|l|}{ Complication } \\
\hline \multicolumn{2}{|l|}{ Pneumothorax* } \\
\hline Small & $28(25.5)$ \\
\hline Large & $7(6.4)$ \\
\hline \multicolumn{2}{|l|}{ Hemorrhage } \\
\hline Mild & $67(60.9)$ \\
\hline Moderate & $1(0.9)$ \\
\hline Localized hematoma & $1(0.9)$ \\
\hline Hemothorax & 0 \\
\hline Hemoptysis & $4(3.6)$ \\
\hline Allergic reaction & 0 \\
\hline Need invasive intervention & 0 \\
\hline
\end{tabular}

*, according to the 2010 British Thoracic Society guidelines, a large pneumothorax is defined as a lung margin to chest wall distance that is larger than $2 \mathrm{~cm}$. CT, computed tomography; PBV, patent blue vital.

Table 3 shows the outcomes of the preoperative CTguided PBV dye localization. Most of the patients ( $\mathrm{N}=110,84 \%)$ received preoperative CT-guided PBV dye localization. Only one technical failure (i.e., an inability to identify the dye intraoperatively) was noted, resulting in a success rate of $99.1 \%$. The procedure related complications were also self-limited, such as asymptomatic pneumothorax ( $\mathrm{N}=35,31.8 \%)$, localized small pulmonary bleeding ( $\mathrm{N}=69$, $62.7 \%)$, and mild hemoptysis ( $\mathrm{N}=4,3.6 \%)$. No allergic or emergent invasive intervention was necessary.

\section{Discussion}

Since 1995, screening with LDCT has helped surgeons to identify small lung nodules. This has led to a substantial reduction in overall mortality from lung cancer because of the greater number of early diagnoses of lung cancer (2). However, with the increasing prevalence of lung cancer screening via LDCT, it has become necessary to determine how to treat the small nodules of early lung cancer. Among the current surgical treatment options for lung cancer, the thoracoscopic approach is an adequate and globally accepted treatment modality that is presented as an alternative to traditional open thoracotomy (20). One systematic literature review (3) demonstrated that the VATS lobectomy is associated with reduced chest tube duration, a shorter hospital stay, and fewer postoperative complications. Additionally, compared with the open lobectomy, the VATS lobectomy demonstrates a reduced systemic recurrence rate and a higher 5 -year survival rate (21).

Currently, lobectomy and systemic lymph node dissection are considered the standard surgical procedures for patients with clinical stage I NSCLC (22). However, sublobar resection is another surgical option, whose features include a low complication rate, short hospitalization duration, and a high preservation of lung function $(23,24)$. In recent studies, sublobar resection has shown equivalent oncological results and survival outcome compared with lobectomy (25-27). Moreover, in 2010, Sugi and colleagues reported a retrospective study of 159 patients, revealing that there were no significant differences among the wedge resection, segmentectomy, and lobectomy approaches in the treatment of small NSCLC (28). Therefore, a limited resection is considered more appropriate in specific patients, such as those with small lung lesions, elderly patients, or those at a high risk of comorbidity. In the present study, we selected patients with small nodules (average diameter: $0.85 \pm 0.40 \mathrm{~cm})$, those with ground-glass predominant nodules, older patients, and patients with an existing extrathoracic malignancy to receive this limited resection.

Along with the development of VATS, there has been an increasing interest in minimally invasive surgery options for the treatment of thoracic disease since the 1990s. At present, there are several strategies to reduce surgical trauma, including: (I) minimizing chest wall trauma by reducing the length of skin incisions or using a thoracoscopic instrument instead of a mechanical retractor or rib spreading; (II) lung-sparing surgery, such as segmentectomy, wedge resection, or sleeve resection, which preserves the pulmonary parenchyma; and (III) nonintubated anesthesia, which reduces airway trauma.

Our team has exerted considerable effort to develop a minimally invasive VATS since 2009. We first focused on developing a nonintubated VATS to reduce the adverse 
effects of tracheal intubation and general anesthesia. Chen et al. (6) revealed that nonintubated thoracoscopic lobectomy lowers complication rates and improves oral intake recovery. Our previous studies have similarly confirmed that nonintubated thoracoscopic lung resection is technically feasible and safe to be a valid alternative for managing patients with pulmonary lesions, even elderly or high-risk patients $(7,13,29)$. Since 2014 , we began utilizing intercostal nerve blocks instead of thoracic epidural anesthesia $(14,19)$. Hung et al. investigated 238 patients, revealing that those who received an intercostal nerve block $(\mathrm{N}=108)$ had a shorter duration of anesthetic induction and a more stable intraoperative hemodynamics (15). Thoracic intercostal nerve blocks provide a regional anesthetic component and exert an anesthetic-sparing effect (30). Moreover, vagal blocks reduce patients' cough reflex, which enables surgeons to complete the procedure smoothly during lung manipulation or dissection of hilar vascular structure. Our well-established protocols using regional blocks and targeted sedation have proven to be safe and effective in various thoracic procedures, including thoracoscopic pneumonectomy (31).

Recently, uniportal thoracoscopic surgery has garnered substantial attention because the procedure is associated with less surgical wound pain, less residual paresthesia, a shorter hospital stay (5) and desirable cosmetic effects. Notably, this procedure can be applied in to most major lung resections (32). Considering the benefits of a single incision technique, combined with the minimal invasiveness that is achieved by avoiding general anesthesia, we have applied this procedure to numerous routine operations. Our results have consistently confirmed that nonintubated uniportal surgery is feasible and safe for the resection of various lung lesions $(9,10)$. Here, we conducted the largest study of nonintubated uniportal VATS for treating primary lung cancer in a single medical institution.

Two key concerns influence the success of uniportal surgery for thoracic malignancies: tumor location and section margin. When performing uniportal VATS, a surgeon must rely on direct vision or instrument palpitation to locate a nodule. In cases of small, deeply located, or predominantly ground-glass nodules, it may be difficult to locate the nodules intraoperatively. In our institution, we have routinely used CT-guided PBV dye localization since 2013 if a surgeon believes it will be too hard to locate the nodule intraoperatively. Lin et al conducted a large retrospective study of 196 patients using preoperative CTguided dye localization to identify pulmonary nodules in
177 uniportal VATS lung resections, and verified that this procedure is efficient and safe (16). Yang et al. reported a case series of 25 consecutive patients who underwent a successful robotic C-arm CT-guided dye localization followed immediately by thoracoscopic surgery in a hybrid operating room; these researchers concluded that this technique has potential to be helpful in future surgery (33). In the present study, 110 patients (84\%) received this procedure, and a highly satisfactory success rate $(99.1 \%)$ was achieved. Only one of the patients was found to have an unidentified lesion during VATS. Moreover, the complications were limited, and included only 35 cases (31.8\%) of asymptomatic pneumothorax, 69 cases $(62.7 \%)$ of small and localized bleeding, and 4 cases (3.6\%) of mild hemoptysis. There were no allergic reactions or emergent situations requiring invasive procedures.

Although there is no conclusive definition of the optimal distance $(34,35)$ between a tumor and the nearest section margin, a margin without malignancy is generally considered to be a crucial factor of recurrence and survival. In uniportal VATS, it can be difficult for surgeons to determine how deep their staple applied to obtain free section margins, especially when patients present with deep and non-palpable nodules. In previous studies, we have used two 2-0 silk anchoring retraction sutures at the proximal and distal side of the nodule $(16,17)$ to extracorporeally retract and lift up the nodule. This practice not only avoids the long instruments that have been conventionally used for lung parenchyma grasping, but also provides sufficient space within which to achieve an adequate section margin. In the present study, all 131 procedures achieved a pathologically complete resection with clear section margin.

In the present study, the conversion rate from uniportal VATS to multiportal VATS was $5.3 \%(\mathrm{~N}=7)$; in other studies, conversion to multi-portal VATS or open thoracotomy has ranged from $0 \%$ to $17.8 \%$, depending on the surgeons' experience and surgical plan (4,17,32,36-39). The reason for conversion is generally attributed to dense pleural adhesion, bleeding, calcified hilar lymph nodes, or unstable patient condition. In the present study, three of the seven conversions were due to an unidentified nodule, which suggests that the preoperative localization of the nodule will be a crucial topic for future research.

Our conversion rate from nonintubated to tracheal intubation with general anesthesia was $0.8 \%$ in the present study. Among experienced surgical teams, the rate of conversion to general anesthesia is generally less than $5 \%$, and is usually conducted because of the presence of pleural 
adhesions, mediastinal movement, bleeding, changes in the surgical plan, hypoxemia, or an intractable cough (40). The conversion rate was very low in this study because of our extensive experience with the procedure.

We acknowledge that this study was limited by its retrospective design and the lack of a control group for comparison. Further detailed investigations that use a prospective controlled design are necessary to more clearly elucidate the impact of this novel technique on perioperative outcomes, postoperative results, and long-term survival.

\section{Conclusions}

We report a large series of clinical cases in which a novel nonintubated uniportal thoracoscopic surgery was performed in the treatment of early primary NSCLC. This minimally invasive thoracic surgery is a feasible, effective, and safe option for the treatment of thoracic malignancy in selected patients.

\section{Acknowledgements}

Funding: This study was supported by research grants from Taiwan Lung Foundation and National Taiwan University Hospital, Taipei, Taiwan (NTUH-MS-419 to JS Chen).

\section{Footnote}

Conflicts of Interest: The authors have no conflicts of interest to declare.

Ethical Statement: Approval for this study was granted by the National Taiwan University Hospital Research Ethics Committee (project approval number: 201706092RINA).

\section{References}

1. Alberg AJ, Brock MV, Ford JG, et al. Epidemiology of lung cancer: Diagnosis and management of lung cancer, 3rd ed: American College of Chest Physicians evidence-based clinical practice guidelines. Chest 2013;143:e1S-e29S.

2. National Lung Screening Trial Research Team, Aberle DR, Adams AM, et al. Reduced lung-cancer mortality with low-dose computed tomographic screening. N Engl J Med 2011;365:395-409.

3. Whitson BA, Groth SS, Duval SJ, et al. Surgery for earlystage non-small cell lung cancer: a systematic review of the video-assisted thoracoscopic surgery versus thoracotomy approaches to lobectomy. Ann Thorac Surg 2008;86:200816; discussion 2016-8.

4. Rocco G, Martin-Ucar A, Passera E. Uniportal VATS wedge pulmonary resections. Ann Thorac Surg 2004;77:726-8.

5. Jutley RS, Khalil MW, Rocco G. Uniportal vs standard three-port VATS technique for spontaneous pneumothorax: comparison of post-operative pain and residual paraesthesia. Eur J Cardiothorac Surg 2005;28:43-6.

6. Chen JS, Cheng YJ, Hung MH, et al. Nonintubated thoracoscopic lobectomy for lung cancer. Ann Surg 2011;254:1038-43.

7. Wu CY, Chen JS, Lin YS, et al. Feasibility and safety of nonintubated thoracoscopic lobectomy for geriatric lung cancer patients. Ann Thorac Surg 2013;95:405-11.

8. Hung MH, Hsu HH, Chen KC, et al. Nonintubated thoracoscopic anatomical segmentectomy for lung tumors. Ann Thorac Surg 2013;96:1209-15.

9. Hung MH, Cheng YJ, Chan KC, et al. Nonintubated uniportal thoracoscopic surgery for peripheral lung nodules. Ann Thorac Surg 2014;98:1998-2003.

10. Hung WT, Hsu HH, Hung MH, et al. Nonintubated uniportal thoracoscopic surgery for resection of lung lesions. J Thorac Dis 2016;8:S242-50.

11. Hung MH, Cheng YJ, Hsu HH, et al. Nonintubated uniportal thoracoscopic segmentectomy for lung cancer. J Thorac Cardiovasc Surg 2014;148:e234-5.

12. Travis WD, Brambilla E, Nicholson AG, et al. The 2015 World Health Organization Classification of Lung Tumors: Impact of Genetic, Clinical and Radiologic Advances Since the 2004 Classification. J Thorac Oncol 2015;10:1243-60.

13. Tseng YD, Cheng YJ, Hung MH, et al. Nonintubated needlescopic video-assisted thoracic surgery for management of peripheral lung nodules. Ann Thorac Surg 2012;93:1049-54.

14. Hung MH, Hsu HH, Chan KC, et al. Non-intubated thoracoscopic surgery using internal intercostal nerve block, vagal block and targeted sedation. Eur J Cardiothorac Surg 2014;46:620-5.

15. Hung MH, Chan KC, Liu YJ, et al. Nonintubated thoracoscopic lobectomy for lung cancer using epidural anesthesia and intercostal blockade: a retrospective cohort study of 238 cases. Medicine (Baltimore) 2015;94:e727.

16. Lin MW, Tseng YH, Lee YF, et al. Computed tomography-guided patent blue vital dye localization of pulmonary nodules in uniportal thoracoscopy. J Thorac Cardiovasc Surg 2016;152:535-44.e2.

17. Lee SK, Son BS, Ahn HY, et al. Single-incision thoracoscopic surgery using an anchoring suture of the 
lung parenchyma for two-directional traction. Ann Thorac Surg 2014;97:e89-91.

18. Tsai TM, Lin MW, Hsu HH, et al. Surgical technique of nonintubated uniportal thoracoscopic wedge resection of a computed tomography-guided tattooed lesion. Asvide 2017;4:447. Available online: http://www.asvide.com/ manuscripts/1769

19. Chen KC, Cheng YJ, Hung MH, et al. Nonintubated thoracoscopic surgery using regional anesthesia and vagal block and targeted sedation. J Thorac Dis 2014;6:31-6.

20. Yang SM, Hsu HH, Chen JS. Recent advances in surgical management of early lung cancer. J Formos Med Assoc 2017. [Epub ahead of print].

21. Yan TD, Black D, Bannon PG, et al. Systematic review and meta-analysis of randomized and nonrandomized trials on safety and efficacy of video-assisted thoracic surgery lobectomy for early-stage non-small-cell lung cancer. J Clin Oncol 2009;27:2553-62.

22. Howington JA, Blum MG, Chang AC, et al. Treatment of stage I and II non-small cell lung cancer: Diagnosis and management of lung cancer, 3rd ed: American College of Chest Physicians evidence-based clinical practice guidelines. Chest 2013;143:e278S-313S.

23. Landreneau RJ, Sugarbaker DJ, Mack MJ, et al. Wedge resection versus lobectomy for stage I (T1 N0 M0) non-smallcell lung cancer. J Thorac Cardiovasc Surg 1997;113:691-8; discussion 698-700.

24. Keenan RJ, Landreneau RJ, Maley RH Jr, et al. Segmental resection spares pulmonary function in patients with stage I lung cancer. Ann Thorac Surg 2004;78:228-33; discussion 228-33.

25. Kates M, Swanson S, Wisnivesky JP. Survival following lobectomy and limited resection for the treatment of stage I non-small cell lung cancer $<=1 \mathrm{~cm}$ in size: a review of SEER data. Chest 2011;139:491-6.

26. Altorki NK, Yip R, Hanaoka T, et al. Sublobar resection is equivalent to lobectomy for clinical stage $1 \mathrm{~A}$ lung cancer in solid nodules. J Thorac Cardiovasc Surg 2014;147:75462; Discussion 762-4.

27. Yano M, Yoshida J, Koike T, et al. Survival of 1737 lobectomy-tolerable patients who underwent limited resection for cStage IA non-small-cell lung cancer. Eur J Cardiothorac Surg 2015;47:135-42.

28. Sugi K, Kobayashi S, Sudou M, et al. Long-term prognosis of video-assisted limited surgery for early lung cancer. Eur J Cardiothorac Surg 2010;37:456-60.

29. Chen KC, Cheng YJ, Hung MH, et al. Nonintubated thoracoscopic lung resection: a 3 -year experience with 285 cases in a single institution. J Thorac Dis 2012;4:347-51.

30. Wang ML, Hung MH, Chan KC, et al. Intraoperative multiple intercostal nerve blocks exert anesthetic-sparing effect: A retrospective study on the effect-site concentration of propofol infusion in nonintubated thoracoscopic surgery. Acta Anaesthesiol Taiwan 2016;54:77-80.

31. Hung WT, Liao HC, Cheng YJ, et al. Nonintubated Thoracoscopic Pneumonectomy for Bullous Emphysema. Ann Thorac Surg 2016;102:e353-5.

32. Gonzalez-Rivas D, Paradela M, Fernandez R, et al. Uniportal video-assisted thoracoscopic lobectomy: two years of experience. Ann Thorac Surg 2013;95:426-32.

33. Yang SM, Ko WC, Lin MW, et al. Image-guided thoracoscopic surgery with dye localization in a hybrid operating room. J Thorac Dis 2016;8:S681-S689.

34. Mohiuddin K, Haneuse S, Sofer T, et al. Relationship between margin distance and local recurrence among patients undergoing wedge resection for small $(</=2 \mathrm{~cm})$ non-small cell lung cancer. J Thorac Cardiovasc Surg 2014;147:1169-75; discussion 1175-7.

35. Maurizi G, D'Andrilli A, Ciccone AM, et al. Margin Distance Does Not Influence Recurrence and Survival After Wedge Resection for Lung Cancer. Ann Thorac Surg 2015;100:918-24; discussion 924-5.

36. Liu CY, Lin CS, Shih CH, et al. Single-port video-assisted thoracoscopic surgery for lung cancer. J Thorac Dis 2014;6:14-21.

37. Hsu PK, Lin WC, Chang YC, et al. Multiinstitutional analysis of single-port video-assisted thoracoscopic anatomical resection for primary lung cancer. Ann Thorac Surg 2015;99:1739-44.

38. Hirai K, Takeuchi S, Usuda J. Single-port video-assisted thoracic surgery for early lung cancer: initial experience in Japan. J Thorac Dis 2016;8:S344-50.

39. Heo W, Kang DK, Min HK, et al. Feasibility and Safety of Single-Port Video-Assisted Thoracic Surgery for Primary Lung Cancer. Korean J Thorac Cardiovasc Surg 2017;50:190-6.

40. Gonzalez-Rivas D, Aymerich H, Bonome C, et al. From Open Operations to Nonintubated Uniportal VideoAssisted Thoracoscopic Lobectomy: Minimizing the Trauma to the Patient. Ann Thorac Surg 2015;100:2003-5.

doi: 10.21037/jovs.2017.08.21

Cite this article as: Tsai TM, Lin MW, Hsu HH, Chen JS. Nonintubated uniportal thoracoscopic wedge resection for early lung cancer. J Vis Surg 2017;3:155. 